\title{
GROUPS WITH ONE DEFINING RELATOR ${ }^{1}$
}

\author{
GILBERT BAUMSLAG 2 \\ (received 26 August 1964)
}

1

The present day theory of finite groups might be regarded as the outgrowth of the algebraic theory of equations. In much the same way one might consider the modern theory of infinite groups as stemming from late nineteenth century topology. The groups that crop up in topology are of a particularly simple type in that they are both finitely generated and finitely related. This means that every element in such a group can be expressed in terms of a finite number of elements and their inverses and every relation is an algebraic consequence of a finite number of relations between these elements. In other words the legacy of topology to group theory is the estate of finitely presented groups. This talk is concerned with the seemingly simplest of the finitely presented groups, the so-called groups with a single defining relator.

It may be as well to repeat and recall some relevant definitions.

Suppose that $G$ is a group and that $X$ is a (possibly empty) subset of $G$. We say that $X$ generates $G$ if for each element $g$ in $G$ we can find $x_{1}, \cdots, x_{n} \in X$ such that

$$
g=x_{1}^{\varepsilon_{1}} \cdots x_{n}^{\varepsilon_{n}} \quad\left(\varepsilon_{i}= \pm 1\right)
$$

by convention the empty set generates the unit group. Naturally enough if $X$ generates $G$ we shall often refer to $X$ as a set of generators of $G$. This notion leads to a rough classification of groups via the cardinality of their generating sets. Thus we say $G$ is an $n$-generator group if it can be generated by a set of cardinality $n$. If $n$ can be chosen finite we say $G$ is finitely generated.

The simplest finitely generated groups are the finitely generated free

1 Invited talk given at the Annual Meeting of the Australian Mathematical Society held at the University of Adelaide in May, 1964.

2 Acknowledgement. The author gratefully acknowlcages support from the National Science Foundation, Grant GP 27. 
groups. We shall use them as a frame of reference with which to compare other finitely generated groups.

The customary way of defining free groups is by means of a universal mapping property. Our definition here is the old-fashioned one in terms of generating sets whose elements have little interaction with each other these are the free sets of generators. To be more explicit, suppose $X$ generates the group $G$. In expressing an element $g \in G(g \neq 1)$ as a power product of the generators $x$ in $X$ (cf. (1)) we can obviously avoid "useless" segments

$$
x_{i}^{\varepsilon_{i}} x_{i+1}^{\varepsilon_{i+1}}
$$

where $x_{i}=x_{i+1}$ and $\varepsilon_{i}+\varepsilon_{i+1}=0$. Let us call the right-hand side of (1) a reduced $X$-product if it does not contain any segments of type (2); the point made above is that every element $g \in G(g \neq 1)$ can be expressed as a reduced $X$-product. If every element $g \in G(g \neq 1)$ can be expressed as a reduced $X$-product in precisely one way, we shall say that $X$ freely generates $G$. A group $G$ is then free if it can be freely generated by some set $X ; X$ is then a free set of generators of $G$.

The cardinality of a free set of generators of a free group turns out to be an invariant (i.e. two sets of free generators have the same cardinality), the rank of the free group in question. It is not difficult to show that free groups of every rank exist; in particular one has then free groups of every finite rank.

I spoke earlier of a universal mapping property. Indeed if $X$ freely generates a group $G$ then it follows easily that for every group $H$ and every mapping $\theta$ of $X$ into $H$ there exists a (unique) homomorphism $\phi$ of $G$ into $H$ which coincides with $\theta$ on $X$. In particular, if $H$ is an n-generator group then we can "present" $H$ as a factor group of a free group $G$ of rank $n$ :

$$
G / R \cong H .
$$

If $n$ is finite, then the isomorphism (3) relates $H$, via a normal subgroup $R$, to a finitely generated free group $G$. But, from our point of view, finitely generated free groups are the simplest finitely generated groups. Therefore the "size" of $R$ measures the deviation from freeness of $H$ and is therefore a determining factor in the complexity of $H$.

The intersection of a family of normal subgroups of a group is again a normal subgroup. Thus if $S$ is a subset of a group $G$ there is a unique minimal normal subgroup of $G$ containing $S$, namely the intersection of all normal subgroups of $G$ containing $S$. We denote this subgroup of $G$ by $\langle S\rangle$ 
and term it the normal closure of $S$ (in $G$ ). It is easy to verify that $\langle S\rangle$ is simply the group generated by the conjugates $g^{-1} s g$ of the elements $s \in S$ by the elements $g \in G$.

Suppose now that we represent a given group $H$ in the form (3) as a factor group of a free group $G$ by a normal subgroup $R$. We shall say that $H$ is defined by the relators $r_{1}, r_{2}, \cdots, r_{m}$ if

$$
R=\left\langle r_{1}, \cdots, r_{m}\right\rangle
$$

If $m$ can be chosen finite we shall say that $H$ is finitely related. The magnitude of $m$ enables us to attach a (vague, perhaps) meaning as to the size of $R-$ the smaller $m$ can be chosen the "smaller" is $R$ and the nearer to a free group is $H$.

We say that the group $H$ is finitely presented if it can be written in the form (3) with $G$ a finitely generated free group and $R$ the normal closure of a finite set of elements of $G$. If $G$ is freely generated by $x_{1}, x_{2}, \cdots, x_{n}$ and $R$ is the normal closure of $r_{1}, r_{2}, \cdots, r_{m}$, then we shall often write

$$
H=\left(x_{1}, \cdots, x_{n} ; r_{1}, \cdots, r_{m}\right) .
$$

Notice also that it is easy to give a meaning to the right hand side of (4) without there being any prior connections with a group $H$, namely simply by putting

$$
\left(x_{1}, \cdots, x_{n} ; r_{1}, \cdots, r_{m}\right)=G / R
$$

where $G$ is the free group freely generated by $x_{1}, \cdots, x_{n}$ and

$$
R=\left\langle r_{1}, \cdots, r_{m}\right\rangle \text {. }
$$

We shall call $H$ a group with one defining relator if we can write it in the form

$$
H=\left(x_{1}, \cdots, x_{n} ; r\right) \quad(n<\infty) .
$$

So by the remarks we have been at great pains to make, groups with a single defining relator should be very near to finitely generated free groups and indeed should then have many of their properties.

\section{4}

The simplest groups with a single defining relator are the finite cyclic groups $Z_{k}$ of order $k$ :

$$
Z_{k}=\left(x ; x^{k}\right) \quad(k=1,2, \cdots) .
$$

However the first groups with a single defining relator which underwent 
intensive study were the fundamental groups of orientable, two-dimensional surfaces. Now orientable surfaces in two dimensions are of a particularly simple type - if the genus $k$ is prescribed then such a surface is homeomorphic to a sphere with $k$ handles. The fundamental group $F_{k}$ of sucl: a surface of genus $k$ is not quite so simple despite the patent simplicity of its presentation

$$
F_{k}=\left(a_{1}, b_{1}, \cdots, a_{k}, b_{k} ; a_{1}^{-1} b_{1}^{-1} a_{1} b_{1} \cdots a_{k}^{-1} b_{k}^{-1} a_{k} b_{k}\right)
$$

$(k=1,2, \cdots)$. Many of the secrets of present day topology are hidden in its structural vagaries; notwithstanding this fact we shall still show that $F_{k}$ is in many ways nearly free.

One of the most extraordinary features of infinite group theory is the fact that many problems involving finitely presented groups do not admit an algorithmic solution (cf. e.g. Rabin [1], Baumslag, Boone and B. H. Neumann [2]). I shall contrast this situation with the fact that many of these same problems do have algorithmic solutions when restricted to groups with a single defining relator.

The most celebrated problem of this type is the "word problem". To explain what this means let $H$ be a finitely presented group with presentation

$$
H=\left(x_{1}, x_{2}, \cdots, x_{n} ; r_{1}, \cdots, r_{m}\right) \quad(n<\infty, m<\infty)
$$

and suppose $G$ is the free group freely generated by $x_{1}, \cdots, x_{n}$ and $R=\left\langle r_{1}, \cdots, r_{m}\right\rangle$. The word problem for $H$ may then be put into the following form: Does there exist an algorithm whereby one can determine whether any element $g$ in $G$ is contained in $R$. P. S. Novikov [3] was first to produce a finitely presented group with an unsolvable word problem i.e. no such algorithm exists. For free groups the uniqueness of reduced $X$ products makes the solution of the word problem a triviality. However for groups with a single relation it is by no means straightforward, the remarkable solution being due to W. Magnus [4].

THEOREM 1. (Magnus) Every group with a single defining relator has a solvable word problem.

Of fundamental importance in the proof of this theorem is the so-called Dehn-Magnus Freiheitssatz, which was formulated by Max Dehn for the fundamental groups $F_{k}$, and proved explicitly by Magnus [5]. To be precise we have the

THEOREM 2. (Magnus) Let $H$ be a group with a single defining relator 


$$
H=\left(x_{1}, \cdots, x_{n} ; r\right) .
$$

Suppose that the first letter in (the reduced $\left\{x_{1}, \cdots, x_{n}\right\}$-product for) $r$ is not the inverse of the last letter in $r$ and that every $x_{i}$ appears in (the reduced $\left\{x_{1}, \cdots, x_{n}\right\}$-product of) $r$. Then every proper subset of $\left\{x_{1}, \cdots, x_{n}\right\}$ freely generates a free group modulo the normal closure of $r$.

Notice that both Theorem 1 and Theorem 2 are in keeping with the previously publicised and promised niceness of groups with a single defining relator. But there is more of a similar nature to come. Firstly one might observe that there is no general and effective procedure whereby one can determine whether any finitely presented group is free (cf. O. Rabin [1]). However for groups with a single defining relator we have the following theorem of J. H. C. Whitehead [6] (cf. also E. S. Rapaport [7]).

THEOREM 3. (J. H. C. Whitehead) There is a general and effective procedure whereby one can determine whether a group with a single defining relator is free.

Secondly, although the corresponding problem for finitely presented groups has a negative solution (cf. e.g. [1]), the following theorem for groups with a single defining relator holds.

Theorem 4. (Karass, Magnus and Solitar [8]) There is a general and effective procedure whereby one can determine whether any group with a single defining relator is torsion-free.

This theorem has recently been successfully exploited by E. S. Rapaport [9] to settle a question of Papakyriakopolous connected with the Poincaré conjecture.

Thirdly the following recent (and as yet unpublished) theorem contrasts favourably with the corresponding negative result for finitely presented groups (cf. [10]):

Theorem 5. (G. Baumslag and T. Taylor) There is a general and effective procedure whereby one can determine whether any group with a single defining relator has a non-trivial centre.

\section{6}

Let $P$ be a property pertaining to groups. Then a group $G$ has the property $P$ resi lally (i.e. the property $\left.{ }_{R} P\right)$ if for each $g \in G(g \neq 1)$

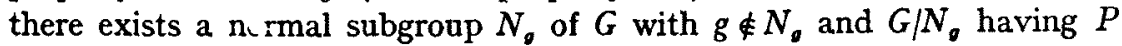
(P. Hall $[11])$.

If $P$ stands for any one of the properties: torsion-free-nilpotence, being a finite $p$-group and finiteness, then free groups are residually $P$ (cf. [12]). Of course one cannot expect that all groups with a single defining 
relator share all these residual properties with free groups. However if one suitably restricts the groups considered one can find analogues of these theorems for free groups; the following four theorems are illustrations of this remark.

THEOREM 6. (G. Baumslag [13]) The group

$$
H=\left(x_{1}, \cdots, x_{n}, y_{1}, \cdots, y_{m} ; u\left(x_{1}, \cdots, x_{n}\right) v\left(y_{1}, \cdots, y_{m}\right)^{-1}\right) \text {, }
$$

where $u\left(x_{1}, \cdots, x_{n}\right)$ and $v\left(y_{1}, \cdots, y_{m}\right)$ lie respectively in the subgroup generated by $x_{1}, \cdots, x_{n}$ and the subgroup generated by $y_{1}, \cdots, y_{m}$, is residually finite.

Theorem 7. (G. Higman [14]) Let $H$ be the group defined in Theorem 6. If neither $u\left(x_{1}, \cdots, x_{n}\right)$ nor $v\left(y_{1}, \cdots, y_{m}\right)$ are proper powers in the subgroups generated respectively by $x_{1}, \cdots, x_{n}$ and $y_{1}, \cdots, y_{m}$, then $H$ is residually a finite p-group for every prime $p$.

Whether the group $H$ in Theorem 7 is residually torsion-free-nilpotent is unknown. In this direction one has

Theorem 8. (G. Baumslag [15]) Let $l$ be a positive integer and let

$$
J=\left(x_{1}, \cdots, x_{n}, x ; x^{l} u\left(x_{1}, \cdots, x_{n}\right)^{-1}\right) \text {. }
$$

If $u\left(x_{1}, \cdots, x_{n}\right)$ is not a proper power in the subgroup generated by $x_{1}, \cdots, x_{n}$ then $J$ is residually torsion-free-nilpotent.

One might term a group $P$ pseudofree if the intersection of the terms $P_{\text {o }}$ of its lower central series is 1 and there exists a free group $G$ such that

$$
P / P_{\mathrm{o}} \cong G / G_{\mathrm{o}}
$$

for $c=1,2, \cdots$. Theorem 8 provides examples of groups with one defining relator which are pseudofree but not free. For example the group

$$
\left(x_{1}, x_{2}, x ; x^{5} x_{2}^{-3} x_{1}^{-2}\right)
$$

is not free, but pseudofree.

Finally one has the following specialisation of Theorem 7 .

TheOREM 9. (G. Baumslag [16]) Let $H$ be as in Theorem 7 and suppose $n=m$. If the isomorphism of the subgroup generated by $x_{1}, \cdots, x_{n}$ onto the subgroup generated by $y_{1}, \cdots, y_{m}$ defined by $x_{i} \rightarrow y_{i}(i=1, \cdots, n)$ takes $u\left(x_{1}, \cdots, x_{n}\right)$ onto $v\left(y_{1}, \cdots, y_{m}\right)$ then $H$ is residually free.

It is not difficult to prove that the fundamental groups $F_{k}$ are subgroups of a group satisfying the hypothesis of Theorem $9(k>1)$. Since subgroups of residually free groups are residually free it follows that the $F_{k}$ are residually free, substantiating a remark made earlier. 
In $1950 \mathrm{~B}$. H. Neumann [17] constructed a finitely generated group which is isomorphic to one of its proper quotient groups. Later G. Higman [18] even constructed a finitely presented group with this property. In a way groups of this type must be complicated and for a long time it was commonly believed that groups with one defining relator cannot behave in this fashion. Indeed finitely generated free groups $G$ are Hopf (i.e. if $G / R \cong G$, then $R=1$ ) since finitely generated residually finite groups are always Hopf (A. I. Mal'cev [19]).

However

Theorem 10. (G. Baumslag and D. Solitar [20]) The group

$$
\left(a, b ; a^{-1} b^{2} a b^{-3}\right)
$$

is isomorphic to a proper quotient group of itself.

So groups with one defining relator are not quite as simple as has been suggested here. Curiously enough G. Higman [21] has quite remarkably characterised the finitely generated subgroups of finitely presented groups. No such characterisation exists for the finitely generated subgroups of groups with one defining relator. Here almost nothing is known. For example the Klein four-group is not a subgroup of a group with one defining relator. One might however ask just which abelian groups are subgroups of a group with one defining relator. I would like to put on record some related conjectures:

The additive group of rationals is not a subgroup of a group with one defining relator.

A simple subgroup of a group with one defining relator is of prime order.

Wilhelm Magnus has tantalisingly, albeit tentatively, suggested that every knot group (cf. [22]) is a subgroup of a group with one defining relator! Be that as it may, groups with one defining relator offer a host of exciting possibilities to the researcher and further understanding of them will increase understanding of finitely presented groups in general.

\section{References}

[1] M. O. Rabin, Recursive unsolvability of group theoretic problems. Annals Math. 67 (1958), 172-194.

[2] G. Baumslag, W. W. Boone and B. H. Neumann, Some unsolvable problems about elements and subgroups of groups. Math. Scand. 7 (1959), 191-201.

[3] P. S. Novikov, On the algorithmic unsolvability of the identity problem, Trudy Mat. Inst. Steklov No. 44 (1955).

[4] W. Magnus, Uber diskontinuierliche Gruppen mit einer definierenden Relation (Der Freiheitssatz), J. reine angew. Math. 163 (1930), 141-165.

[5] W. Magnus, Das Identitätsproblem für Gruppen mit einer definierenden Relation, Math. Ann. 106 (1932), 295-307. 
[6] J. H. C. Whitehead, On certain sets of elements in a free group, Proc. London Math. Soc. 41 (1936), 48-56.

[7] E. S. Rapaport, On free groups and their automorphisms, Acta Math. 99 (1958), 139-163.

[8] A. Karass, W. Magnus and D. Solitar, Elements of finite order in groups with a single defining relation, Comm. Pure Appl. Math. 13 (1960) 57-66.

[9] E. S. Rapaport, Proof of a conjecture of Papakyriakopoulos, Annals of Maths 79 (1964) $506-513$

[10] G. Baumslag and T. Taylor, The centre of groups with one defining relator. To be submitted to Math. Annalen.

[11] P. Hall, The splitting properties of relatively free groups, Proc. London Math. Soc. 4 (1954), 343-356.

[12] K. W. Gruenberg, Redisual properties of infinite soluble groups, Proc. London Math Soc. 7 (1957), $29-62$.

[13] G. Baumslag, On the residual finiteness of generalised free products of nilpotent groups, Trans. American Math. Soc. 106 (1963), 193-209.

[14] G. Higman, Unpublished.

[15] G. Baumslag, The residual torsion-free-nilpotence of certain groups with one defining relation. To be submitted to Journal of Algebra.

[16] G. Baumslag, On generalised free products, Math. Zeitschr. 78 (1962), 423-488.

[17] B. H. Neumann, A two-generator group isomorphic to a proper factor group. J. London Math. Soc. 25 (1950), 247-248.

[18] G. Higman, A finitely related group with an isomorphic proper factor group, J. London Math. Soc. 26 (1951), 59-61.

[19] A. I. Mal'cev, On isomorphic matrix representations of infinite groups, Mat. Sb. (N.S.) 8 (50) (1940), 405-421.

[20] G. Baumslag and D. Solitar, Some two-generator one-relator non-hopfian groups, Bull. American Math. Soc. 68 (1962), 199-201.

[21] G. Higman, Subgroups of finitely presented groups, Proc. Royal Soc. A, 262 (1961), 455-475.

[22] Crowell and R. H. Fox, Introduction to knot theory. Ginn and Co., New York (1963).

City University of New York, 33 West 42nd Street,

New York City. 